\title{
Strategy on Disaster Recovery Management based on Graph Theory Concepts
}

\author{
Jerlin Seles M, U. Mary
}

\begin{abstract}
The COVID-19 pandemic has asserted major baseline facts from disaster anthropology during the last three decades. Resilience could be based on the solution to the question: "What is the maximum amount of destruction, if any, that the graph (a network) can sustain while ensuring that at least one of each technology type remains and that the remaining induced subgraph is properly colored?" The concept of a graph's Chromatic Core Subgraph is a solution to the stated problem. In this paper, the pandemic graphs and certain sequential graphs are developed. For these graphs, the Chromatic core subgraph is obtained. The results of the pandemic graphs' Chromatic core subgraph are used to develop a disaster recovery strategy for the COVID-19 pandemic.
\end{abstract}

Keywords: COVID-19, Directed graphs, Disaster Recovery Plan, Jaco-type graph, Pandemic.

\section{INTRODUCTION}

For general definitions on graphs and digraphs [1]-[4]. The number of edges that touch the vertex, $v$ of the graph $G$ is known as the degree of that vertex, denoted $\operatorname{deg}(v)$. A functional graph is a digraph that may be built by a function mapping $\{1,2,3, \ldots, n\}$ onto itself. Every vertex in such graphs has an outdegree of one. The definition of a linear Jaco graph [5] (all Jaco graphs for that matter) incorporates an integer valued linear function. The function value of the vertex subscript $f(i)$ of vertex $v_{i}$ determines the total vertex degree $\operatorname{deg}\left(v_{i}\right)$. Observe that the most theoretical underpinnings of any graph $G$ is its chromatic number. The Chromatic number [4] of a graph $G$, given by $\chi(G)$, is the minimum number of colors required to color the vertices such that no monochromatic edges exist. This style of coloration is referred to as proper coloring. Coloring planar graphs which can be formed from a map was one of the early applications of the graph coloring subject. It further resulted in the famous Four Colors theorem, which stipulates that planar graph can be colored properly with four shades. The field of graph coloring problems and its applicability has now become span less.The idea of Chromatic Core Subgraph [6], abbreviated as CCS, is obtained in this study for two non-negative, non-decreasing integer sequences known as Jaco-type graphs.

Manuscript received on September 09, 2021.

Revised Manuscript received on September 16, 2021.

Manuscript published on September 30, 2021.

* Correspondence Author

Jerlin Seles $\mathbf{M}^{*}$, Department of Mathematics, Nirmala College for Women, Coimbatore (Tamil Nadu), India. Email: selesjerlin21@gmail.com

Dr. U. Mary, Department of Mathematics, Nirmala College for Women, Coimbatore (Tamil Nadu), India. Email: marycbe@gmail.com

(c) The Authors. Published by Blue Eyes Intelligence Engineering and Sciences Publication (BEIESP). This is an open access article under the CC BY-NC-ND license (http://creativecommons.org/licenses/by-nc-nd/4.0/)
As an application to this concept, the pandemic graphs are created using statistical analysis of data collected on COVID-19 patients in India. The recovery technique $[7,8]$ is designed based on the chromatic core subgraph of pandemic graphs.

\section{CHROMATIC CORE SUBGRAPH OF JACO- TYPE GRAPHS}

The earliest research on this topic focused on Clique Invariants of Jaco-type graphs as in [9]. These graphs are graphical representations of integer sequences. These types of graphs were first introduced in [10]. The Chromatic Core Subgraph of Jaco-type graphs for Sequences and FibonacciSequence are obtained in this section.

Definition 2.1. [6] For a finite, undirected simple graph $G$ of order $v(G)=n \geq 1$, a chromatic core subgraph $H$ is a smallest induced subgraph $H$ (smallest in respect of $\operatorname{si}(H)$ ) such that, $\chi(G)=\chi(H)$.

Definition 2.2. A finite, undirected simple graph's vertex is called as the graph's core vertex $v_{\text {ccs }}$, if it satisfies the following conditions:

- $\operatorname{deg}\left(v_{c c s}\right)=\Delta(G)$

- $v_{c c s}$ forms edge with the end vertex of the graph $G$.

\section{A. Results on Jaco-type Graphs for Sequences}

The infinite Jaco-type graph [9] for Sequences $J_{\infty}\left(s_{1}\right)$ is defined as a graph with vertex set $V\left(J_{\infty}\left(s_{1}\right)\right)=\left\{v_{i}: i \in \mathrm{N}\right\}$ and arc set $A\left(J_{\infty}\left(s_{1}\right)\right)=\left\{\left(v_{i}, v_{j}\right): i, j \in \mathrm{N}, i<j\right\}$ such that $\left(v_{i}, v_{j}\right) \in A\left(J_{\infty}\left(s_{1}\right)\right)$ if and only if $2 i \geq j$. It's worth noting that in this family, a finite Jaco-type graph $J_{n}\left(s_{1}\right)$ is generated from $J_{\infty}\left(s_{1}\right)$ by discarding all vertices $v_{k}, \forall k>n$ (with incident arcs).

Fig. 1 depicts a Jaco-type graph $J_{8}\left(s_{1}\right)$

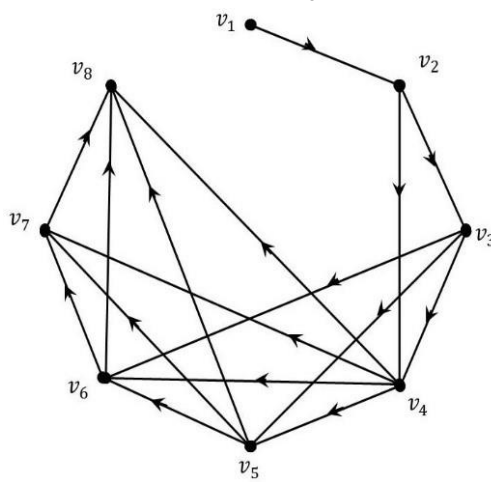

Fig. 1. $J_{8}\left(s_{1}\right)$

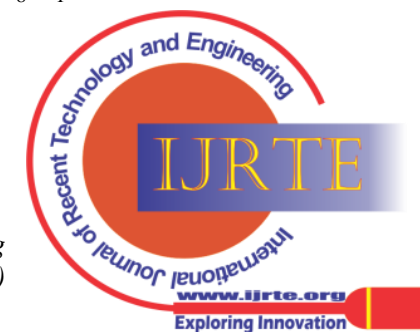




\section{Strategy on Disaster Recovery Management based on Graph Theory Concepts}

Theorem 2.1: The Chromatic Core Subgraph of $J_{n}\left(s_{1}\right)$ is given by $\operatorname{CCS}\left(J_{n}\left(s_{1}\right)\right)=\left\{\begin{array}{l}K_{(n+1) / 2}: n \text { is odd and } n \geq 1 \\ K_{\left(\frac{n}{2}\right)+1}: n \text { is even and } n \geq 2\end{array}\right.$

Proof:

For $J_{1}\left(s_{1}\right)$ we have $K_{1}$.

For $J_{2}\left(s_{1}\right)$ we have $K_{2}$.

For $J_{3}\left(s_{1}\right)$ we have the graph $P_{3}$ with chromatic number, $\chi\left(P_{3}\right)=2$ which is obvious, as any path graph can be colored by two colors.

The graph is deconstructed into its subgraphs in such a way that the graph and its subgraphs have the same chromatic number. In this way, the graph $P_{3}$ is divided into two complete subgraphs $K_{2}, K_{2}$ on vertices $\left(v_{1}, v_{2}\right)$ and $\left(v_{2}, v_{3}\right)$. The chromatic number, $\chi\left(K_{n}\right)$ is generally known to be $n$. In this case, we have the chromatic number of the subgraphs to be two. $K_{2}, K_{3}$. The graph $J_{4}\left(s_{1}\right)$ can be colored using three colors. There is a subgraph $K_{3}$ among the subgraphs of $J_{4}\left(s_{1}\right)$ that may also be colored with three colors. The chromatic core subgraph of $J_{4}\left(s_{1}\right)$ is $K_{3}$ since $\chi J_{4}\left(s_{1}\right)=\chi\left(K_{3}\right)=3$.

The destruction into subgraphs for $J_{5}\left(s_{1}\right)$ corresponds to $K_{2}, K_{3}, K_{3}$. The chromatic core subgraph of $J_{5}\left(s_{1}\right)$ is $K_{3}$ due to the fact that $\chi J_{5}\left(s_{1}\right)=\chi\left(K_{3}\right)=3$.

For $J_{6}\left(s_{1}\right)$ the decomposition into subgraphs corresponds to $K_{2}, K_{3}, K_{4}$. Clearly the result holds for, $J_{l}\left(s_{1}\right), 1 \leq l \leq 6$.

Assume, the result holds for $1 \leq l \leq m$ and without losing generality assume that there exist a core vertex $v_{c c s}$ such that it has the maximum degree of $J_{m}\left(s_{1}\right)$ that is, $\Delta\left(J_{m}\left(s_{1}\right)\right)=\operatorname{deg}\left(v_{c c s}\right)$ and the arc $\left(v_{c c s}, v_{m}\right)$ exists. Also, the arcs $\left(v_{c c s}, v_{c c s+1}\right), \ldots,\left(v_{c c s}, v_{m}\right)$ forms the chromatic core subgraph of $J_{m}\left(s_{1}\right)$. Extend to $J_{m+1}\left(s_{1}\right)$. Clearly, a complete graph is created.

If $m$ is even, then the index of core vertex of vertex.

For the case where $m$ is odd, the argument is the same. As a result of induction, the conclusion follows.

\section{B. Jaco-type Graphs for Fibonacci-Sequences}

The infinite Jaco-type graph corresponding to FibonacciSequence [9] which is also called the Fibonaccian Jaco-type graph $J_{\infty}\left(s_{2}\right)$ is defined by the vertex set $V\left(J_{\infty}\left(s_{2}\right)\right)=\left\{v_{i}: i \in \mathrm{N}\right\} \quad$ and $\operatorname{arc}$ set $A\left(J_{\infty}\left(s_{2}\right)\right)=\left\{\left(v_{i}, v_{j}\right): i, j \in \mathrm{N} ; i<j\right\} \quad$ such that $\left(v_{i}, v_{j}\right) \in A\left(J_{\infty}\left(s_{2}\right)\right)$ if and only if $i+f_{i} \geq j$ where $f_{i}$ is the Fibonacci sequence $f_{0}=0, f_{1}=1, \ldots, f_{n}=f_{n-2}+f_{n-1}: n \in \mathrm{N}$.

Note that, a finite Jaco-type graph $J_{n}\left(s_{2}\right)$ in this family is obtained from $J_{\infty}\left(s_{2}\right)$ by lobbing off all vertices $v_{k}, \forall k>n$.
The destruction into subgraphs for $J_{4}\left(s_{1}\right)$ corresponds to $J_{m}\left(s_{1}\right)=\left(\frac{m}{2}\right)+1=\frac{(m+1)+1}{2}=J_{m+1}\left(s_{1}\right), \quad$ index of core

Fig. 2 depicts a Jaco-type graph $J_{12}\left(s_{2}\right)$

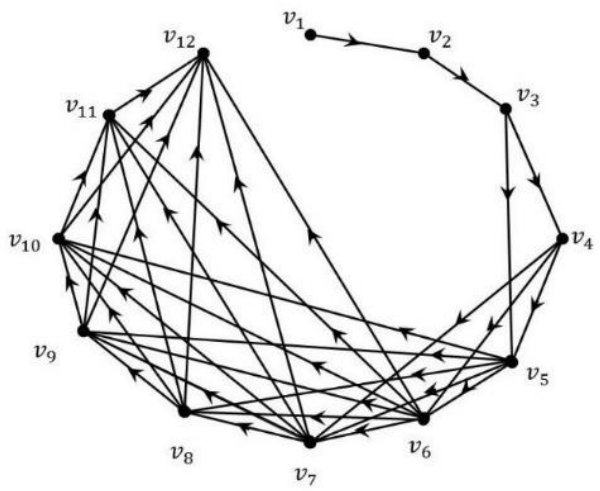

Fig. 2. $J_{12}\left(s_{2}\right)$

Theorem 2.2. The Chromatic Core Subgraph of $J_{n}\left(s_{2}\right)$ is given by $\operatorname{CCS}\left(J_{n}\left(s_{2}\right)\right)=\left\{\begin{array}{l}K_{\frac{(n+1)}{2}}: n \text { is odd and } n \geq 1 \\ K_{2}: n=2 \\ K_{\left(\frac{n}{2}\right)}: n \text { is even and } n \geq 4\end{array}\right.$

Proof.

For $J_{1}\left(s_{2}\right)$ we have $K_{1}$.

For $n=2$ is a special case. Here for the graph $J_{2}\left(s_{2}\right)$ we have $K_{2}$.

For $J_{3}\left(s_{2}\right)$ we have the graph $P_{3}$ with chromatic number, $\chi\left(P_{3}\right)=2$ which is obvious, as any path graph can be colored by two colors.

The graph is deconstructed into its subgraphs in such a way that the graph and its subgraphs have the same chromatic number. In this way, the graph $P_{3}$ is divided into two complete subgraphs $K_{2}, K_{2}$ on vertices $\left(v_{1}, v_{2}\right)$ and $\left(v_{2}, v_{3}\right)$. The chromatic number, $\chi\left(K_{n}\right)$ is generally known to be $n$. In this case, we have the chromatic number of the subgraphs to be two.

The destruction into subgraphs for $J_{4}\left(s_{2}\right)$ corresponds to $K_{2}, K_{2}, K_{2}$. The graph $J_{4}\left(s_{2}\right)$ can be colored using three colors. There is a subgraph $K_{2}$ among the subgraphs of $J_{4}\left(s_{2}\right)$ that may also be colored with three colors. The chromatic core subgraph of $J_{4}\left(s_{2}\right)$ is $K_{2}$ since $\chi J_{4}\left(s_{2}\right)=\chi\left(K_{2}\right)=2$.

The destruction into subgraphs for $J_{5}\left(s_{2}\right)$ corresponds to $K_{2}, K_{2}, K_{3}$. The chromatic core subgraph of $J_{5}\left(s_{2}\right)$ is $K_{3}$ due to the fact that $\chi J_{5}\left(s_{2}\right)=\chi\left(K_{3}\right)=3$.

For $J_{6}\left(s_{2}\right)$ the decomposition into subgraphs corresponds to $K_{2}, K_{2}, K_{3}, K_{3}$.

Clearly the result holds for, $J_{l}\left(s_{2}\right), 1 \leq l \leq 6$. Assume, the result holds for $1 \leq l \leq m$ and without losing generality assume that there exists a core vertex $v_{c c s}$ such a way that it is the vertex of chromatic core subgraph of $J_{m}\left(s_{2}\right)$. Extend to $J_{m+1}\left(s_{2}\right)$.

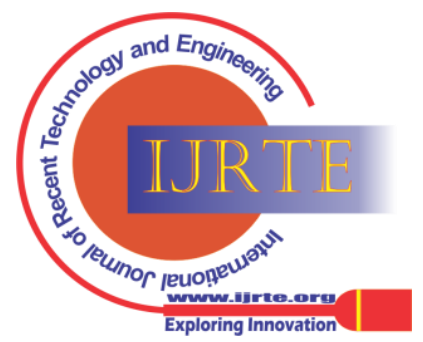


Clearly, a complete graph is created, which is the chromatic core subgraph.

\section{APPLICATION TO CHROMATIC CORE SUBGRAPH}

A disaster is an unanticipated, cataclysmic unfortunate incident that has a massive effect on an organization's or society's ability to cope and causes human, material, financial, or ecological damages that exceed the public's or culture's ability to deal with its own resources. Despite the fact that disasters are most often caused by nature, they can have human origins. Either during or soon after a disaster, damage occurs. The destruction of tangible infrastructure, the disruption of important services, and the effects on livelihoods in the impacted region are typically assessed in metrics (e.g., square metres of housing, kilometres of roads, and so on). As awful as the word "disaster" may seem, there are a multitude of "disasters" that could befall the organization, causing IT system failure or loss of data. A disaster recovery strategy defines how quickly a company can respond to and recover from these catastrophes.

The following three primary factors should be addressed in any disaster recovery plan:

1. Protocols for documenting the proper emergency reaction to a wild fire, natural catastrophe, or any other incident in order to protect people and prevent losses.

2. Standby operation plans should be developed to ensure that valuable data processing operations can continue after the outage.

3. Strategies for restoring the systems quickly after a calamity.

Any technology type (network) can be graphically built up at the vertices of a graph, so the service's eventual extinction as a result of a major disaster can also be graphically depicted. Based on the third major aspect indicated above in the disaster recovery plan, the concept of Chromatic core subgraph is applied in this work as a technique for restoring management systems.

\section{A. Materials and Method}

We characterized and analysed the data set collected from Kaggle on COVID- 19 instances in India between January to May of 2021. Indian states are divided into six Zonal codes which are used as an independent variable. The dependent variables in this study include died and confirmed patients across the state. The Kruskal-Wallis test is used to assess statistically significant variations between groups of an independent variable on a continuous or ordinal dependent variable. In this study, the distribution of Deaths and Confirmed cases among Zone code categories is not normal. As a result, for non-parametric tests, Dunn's Multiple Comparison Test is used as a post hoc. It is one of the least consistent multiple comparison tests that can be used for larger number of comparisons. This test is performed to determine graphical representations of the data. On the basis of the Chromatic core subgraph of graph derived from the COVID-19 data-set, a disaster recovery management technique is devised.

\section{B. Statistical Analysis}

Descriptive statistics, the Kruskal-Wallis test, and the Dunn's post hoc test are used to analyse the acquired data. Predictive analysis software "IBM SPSS Statistics (Version 27)" was used to evaluate the data.

\section{Results using Pandemic graphs}

India's states are considered as an independent variable. Six zonal codes are used to partition the states. The six zones considered are tabulated in Table 1 . The results of the analysis of deceased and infected patients are presented in this section.

Table- I: The Zonal Codes

\begin{tabular}{|c|c|}
\hline Zone Code & Zone Description \\
\hline 1 & Central India \\
\hline 2 & East India \\
\hline 3 & North India \\
\hline 4 & Northeast India \\
\hline 5 & South India \\
\hline 6 & Western India \\
\hline
\end{tabular}

Patients who died from the Corona virus is the first dependent variable, it is analysed using the Kruskal-Wallis test. The Dunn's post hoc test is used to create the graphical representations for this data set, which we refer to as pandemic graphs (Fig. 3). Null hypothesis: The number of patients who have died is distributed evenly across all Zone code groups. Decision: At $0.5 \%$ significance level we reject the null hypothesis. That is, the differences between the variables are statistically significant. In other words, the distribution of dead patients differs by zone code. The pandemic graph created from the data of deceased patients can be colored in four different ways. As illustrated in Fig. 3 , when we decompose the graph, we get two types of subgraphs: $K_{3}$ and $K_{4}$. One of these subgraphs is $K_{4}$, which may also be colored with four distinct colors. As a result, it is the chromatic core subgraph of the graph formed from the data-set of coronavirus-deceased patients.

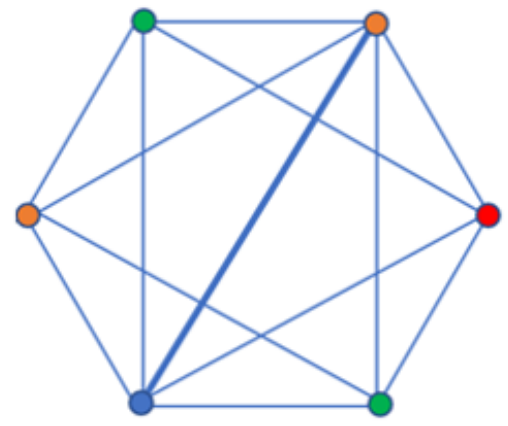

Fig. 3. Graph of coronavirus-deceased patients

Patients infected with the Corona virus is the second dependent variable, and they are similarly analysed using the Kruskal-Wallis test. The graphical representations of pandemic graphs for infected case are generated using the Dunn's post hoc test (See Fig. 4). Null hypothesis: The distribution of infected patients is the same throughout Zone code domains. Decision: At a significance level of 0.5 percent, we reject the null hypothesis. That is, the differences between the variables are statistically significant To put it another way, the distribution of infected patients differs by zone code. The graph generated from infected patients' data depicted in Fig. 4 can be colored with four different colors. When we breakdown the graph, we get two types of subgraphs: $K_{3}$ and $K_{4}$, as shown in Fig. $4 . K_{4}$ is one of these subgraphs, and it can be colored with four different colors.

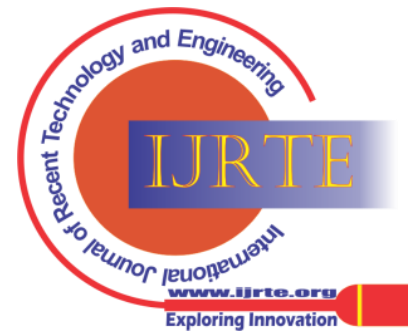




\section{Strategy on Disaster Recovery Management based on Graph Theory Concepts}

As a result, it is the chromatic core subgraph of the graph generated from the data set of coronavirus-infected subjects.

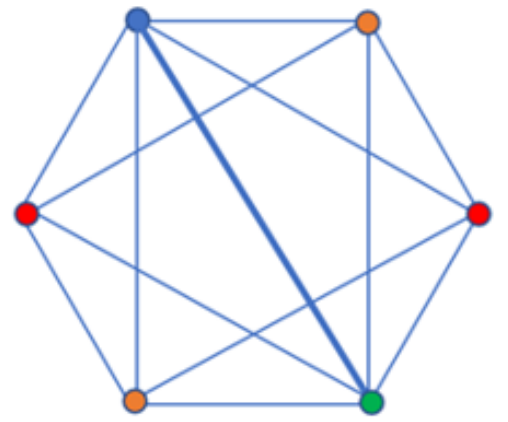

Fig. 4. Graph of coronavirus-infected subjects

\section{DISCUSSION}

Therefore, as result, when a system that can be visually represented is destroyed, its core subgraph may be identified and the system can be recreated. By strengthening the core subgraph, the system can be repaired. In this study, we propose to use this reconstruction technique as a catastrophe recovery strategy.

\section{CONCLUSION}

The idea of Chromatic core subgraphs on pandemic graphs is discussed as an application in this paper. There are also theorems for identifying Chromatic core Subgraphs in Jacotype graphs. Based on the data from the pandemic graphics, a catastrophe recovery strategy is outlined. Many of the number sequences revealed in Sloane's OEIS bring up a lot of possibilities for future investigation.

\section{Problem:}

Investigate the Chromatic core subgraphs of all possible Jaco-type graph orders in terms of:

- Lucas numbers: $L_{0}=2, L_{1}=1, L_{n}=L_{n-1}+$ $L_{n-2}, n \geq 2$

- $\quad$ Sequence of primes: $2,3,5,7,11 \ldots$

- Triangular numbers: $T_{n}=\left(\begin{array}{c}n+1 \\ 2\end{array}\right), n \geq 1$

- Catalan numbers: $C_{n}=\frac{1}{n+1}\left(\begin{array}{c}2 n \\ n\end{array}\right)$

\section{REFERENCES}

1. J. A. Bondy, U. S. R. Murty, Graph Theory with Applications. Macmillan Press, London, 1976.

2. G. Chartrand, L. Lesniak, Graphs and Digraphs. CRC Press, Boca Raton, 2000

3. D. B. West, Introduction to Graph Theory. Pearson Education, 2001.

4. F. Harary, Graph Theory. Narosa Publishing, New Delhi, 2001.

5. J. Kok, "Linear Jaco graphs: A critical review," Journal of Informatics and Mathematical Sciences, vol. 8, 2016, pp. 67-103.

6. J. Kok, N. K. Sudev, M. Jerlin Seles, U. Mary, "On chromatic core subgraph of simple graphs," Contemporary Studies in Discrete Mathematics, vol. 2, 2018, pp. 13-20.

7. J. McDonald, R. Mitra, Movements in Organizational Communication Research, Routledge, New York, 2019.

8. I. Walter Vikas, M. Jerlin Seles, J. Kok, "Experimental study of two organisational development techniques," American International Journal of Research in Humanities, Arts and Social Sciences, vol. 23, 2018, pp. 63-69.

9. M. Jerlin Seles, U. Mary, J. Kok, "A study on clique invariants of Jaco-type graphs," Communications in Mathematics and Applications, vol. 7, 2016, pp. 329-342.

10. J. Kok, N. K. Sudev, K. P. Chithra, U. Mary, "Jaco-type graphs and black energy dissipation," Advances in Pure and Applied Mathematics, vol. 8, 2017, pp. 141-152.

\section{AUTHORS PROFILE}

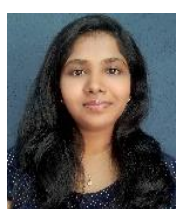

Jerlin Seles $\mathbf{M}$ is a lecturer in statistics at Texila American University's Pre-medical department in Guyana, South America. She is now pursuing her $\mathrm{PhD}$ and has a Master of Philosophy in Graph Theory from Nirmala College for Women as well as a Post-Graduation in Mathematics from PSG College of Art and Science. Bharathiar University awarded her MPhil thesis with high commendation. She is a researcher in the field of mathematics, with a focus on graph theory, who is also interested in diverse applications of graph theory and discrete mathematics in fields such as engineering and social and biological networks. She has experience as a statistician for research papers.

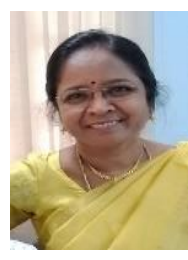

Dr. U. Mary is an Associate Professor and the Head of the PG and Research Department of Mathematics at Nirmala College for Women in Coimbatore, Tamil Nadu, India. She has been teaching mathematics for almost 35 years. Her area of study is graph theory which includes Coloring, Labelling and Topological Indices. She produced 30 M.Phil. Scholars in all and currently advising eight Ph.D. candidates. She has more than 50 articles in internationally recognized journals such as SCOPUS, Elsevier, UGC CARE and others. Also have presented more than ten research papers at major international conferences. She travelled to France and Italy to present a research paper at an international conference.
Published By:

Blue Eyes Intelligence Engineering and Sciences Publication (BEIESP)

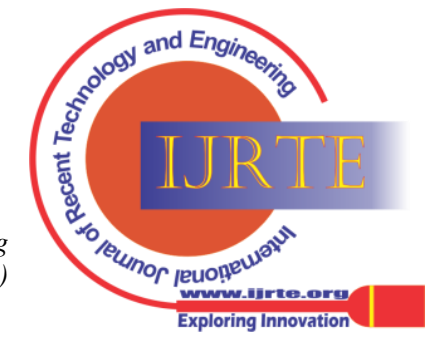

